\title{
CRECIMIENTO INICIAL DE Guazuma crinita \\ TRASPLANTADA A CAMPO ABIERTO CON APLICACION DE TRES DOSIS DE HUMUS DE LOMBRIZ Y A TRES DISTANCIAMIENTOS
}

A mérico Quevedo G. ${ }^{*}$

\section{RESUMEN}

En Pucallpa-Perú, existen vastas áreas deforestadas como producto del uso irracional, principalmente de las especies maderables. Es necesario reforestar urgentemente con uso de técnicas económicas y disponibles. Se realizó un trabajo para determinar el crecimiento inicial de G. crinita en términos de altura y diámetro de la plantación por efecto del trasplante en condiciones de campo abierto usando dosis de 0,1 , y $2 \mathrm{~kg} /$ planta de humus de lombricultura (HL) y tres distancias de siembra de $1 \times 1,2 \times 2$, y $3 \times 3 \mathrm{~m}$, durante 150 días. Se encontró que: i) La velocidad de crecimiento cada 15 días fue de $7 \mathrm{~cm}$ en la altura de G. ermita, por efecto del uso de HL en el trasplante a campo abierto, durante los primeros 150 días, u) G. crinita alcanzó un incremento de $305 \%$ de altura por efecto de la aplicación de $1 \mathrm{~kg} /$ planta de $\mathrm{HL}$, durante 150 días, trasplantadas a campo abierto en un suelo de Pucallpa, iii) el crecimiento de G. crinita en términos de altura y diámetro de plantas fue afectado positivamente por la aplicación de HL y fue significativamente mayor que el testigo $\sin \mathrm{HL}$, iv) a los 150 días después del trasplante a campo abierto y con aplicación de $\mathrm{HL}$, el distanciamiento de siembra de $1 \times 1,2 \times 2,3 \times 3 \mathrm{~cm}$, en $\mathrm{G}$. ermita, no tuvo efecto ni en su altura ni en su diámetro, y v) es suficiente una aplicación $1 \mathrm{~kg} / \mathrm{ha}$ de HL para un buen establecimiento de la plantación de bolaina.

\section{INTRODUCC ION}

En la selva peruana, la búsqueda de técnicas de plantación en campo abierto para recuperar y establecer bosques artificiales, tiene como una de sus restricciones las limitaciones de suelo, tales como acidez, bajos niveles de materia orgánica y de $\mathrm{N}, \mathrm{P}, \mathrm{K}$, y $\mathrm{Mg}$. Consecuentemente, se necesita hacer correcciones de la fertilidad del suelo usando abonos orgánicos en la reforestación con especies de rápido crecimiento.

* Ing. Forestal, M. Sc. en M anejo de Bosques, Jefe del Proyecto Forestal del IIA P U cayali. Pucallpa, Perú. 
U na de las posibilidades para reforestar en condiciones de campo abierto es el uso de humus de lombricultura (HL) como abono en el trasplante y establecimiento de plantaciones forestales. Por lo tanto es necesario conocer el efecto del $\mathrm{HL}$ en el crecimiento de especies de ciclo corto como la Bolaina (G uazuma crinita).

Con el propósito de lograr una técnica de regeneración con especies forestales en suelos degradados de la A mazonía, el objetivo de este trabajo fue determinar el crecimiento inicial de Guazuma crinita en el trasplante con aplicación de tres dosis de $\mathrm{HL}$ y con tres distanciamientos de siembra, en un suelo de Pucallpa.

\section{REVISION BIBLIOGRAFICA}

\section{SILVICULTURA DE LA BOLAINA (Guazuma crinita)}

La Bolaina (G. crinita), en el Perú, se encuentra en la A mazonía y la Costa Norte: A mazonas, Cajamarca, Huánuco, Junín, Lambayeque, Loreto, San M artín, y U cayali (Encarnación, 1983). Es un árbol que al canza una altura de 30 $\mathrm{m}$ y un diámetro de 25 a $50 \mathrm{~cm}$.

Los suelos preferidos de la bolaina son los ricos, con buen drenaje, inundables temporalmente, también tolera suelos pobres con cierta deficiencia en el drenaje, pero es baja la tolerancia a la competencia. Crece en mánchales, asociado con otras especies pioneras como Schizolobiurn sp., Croton sp., Cecropia sp., entre otros. Sufre ataque de grillos que despuntan la yema principal, la misma que conduce a la difurcación del tallo. La técnica de plantación es siembra directa, en envase o a raíz desnuda bajo el sistema de plantación a campo abierto, especial para la combinación con cultivos agrícolas anuales o perennes. Su comportamiento inicial es de rápido crecimiento, formando una pequeña copa en forma globosa a un tercio superior del tronco.

En trabajos realizados sobre regeneración artificial con Guazuma crinita por INFOR-JICA (1985), lograron en el sistema de fajas de enriquecimiento un crecimiento anual de $1.64 \mathrm{~m}$ de altura (faja de $5 \mathrm{~m}$ de ancho), $1.79 \mathrm{~m}$ de altura (faja de $10 \mathrm{~m}$ de ancho) y $2.73 \mathrm{~m}$ de altura (faja de $30 \mathrm{~m}$ de ancho). Se observó que a medida que el ancho de la faja se incrementa, el crecimiento en altura es mayor. Consecuentemente, la plantación a campo abierto tiene mejor comportamiento.

Carrera (1987), en estudios realizados sobre plantaciones forestales y con varias especies, determiné que la bolaina es la especie que más se ha desarrollado tanto 
en altura como en diámetro en el sistema de fajas de enriquecimiento, por lo que es seguro que su crecimiento es mayor en plantaciones a campo abierto, ya que son especies heliófitas, con carácter de pioneras. La bolaina a una edad de 3.4 años, con fajas de, 10 y $30 \mathrm{~m}$ de ancho, tuvieron una altura de $5,8,11.2$ y 13.8 $\mathrm{m}$ de altura, respectivamente, y un diámetro de $3.3,9.9$ y $11.1 \mathrm{~cm}$, respectivamente.

\section{HUMUS DE LOMBRICULTURA (HL) EN LA AMAZONÍA PERUANA}

El HL es el proceso final de la descomposición de la materia orgánica, esto es básicamente la mineralización y resíntesis de las sustancias orgánicas en complejos coloidales amorfos, Las lombrices mineralizan enzimáticamente la materia orgánica en el primer tercio de su aparato digestivo y luego la humifican en la parte posterior del intestino por acción de los microorganismos presentes en esta sección intestinal (Sabac-Chile, 1980)

Los ensayos con aplicación de $\mathrm{HL}$ en suelos degradados de Pucallpa demuestran que tiene un efecto significativo en el rendimiento de hortalizas así como en las propiedades del suelo (Ríos y Rime, 1992; Ríos et al, 1993; Guerra, 1993). También tiene efecto importante en el crecimiento y vigor de especies forestales al sembrar plantones de Cedrela odorata en plantación a campo abierto y en suelo degradado, enriqueciendo el sustrato con $\mathrm{HL}$. Además redujo la mortalidad ante el ataque de Hypsipila sp. vigorizando el planton y favoreciendo el "rebrote" de nuevas yemas, en Pucallpa (Quevedo, 1991).

\section{MATERIALES Y METODOS}

\section{LOCALIZACIÓN Y CARACTERÍSTICAS DEL ÁREA EXPERIMENTAL}

EI estudio se desarrolló en el terreno del CRI-IIAP-U cayali, a $12 \mathrm{~km}$ de la ciudad de Pucallpa, región U cayali. El área presenta topografía plana, con poca vegetación arbustiva, característica de las áreas deforestadas a lo largo de la carretera Pucallpa-Tingo M aría.

Las coordenadas geográficas son 8 23' 31" S, 7034'35" O, y a 162 msnm. El clima es cálido. La temperatura media anual es de 25.7 ㄷ C, la precipitación media anual es de $1324 \mathrm{~mm}$, y la humedad relativa del aire es de 87\%. Según el Sistema Holdrige, la región U cayali se clasifica como Bosque Húmedo Tropical.

Los suelos que predominan en la región son los Ultisoles, ácidos, bajos en contenido de nutrientes y materia orgánica. Las propiedades del suelo del área experimental se muestran en el cuadro 1. 


\section{Cuadro 1}

\section{Propiedades del suelo del área experimental en Pucallpa}

\begin{tabular}{|l|l|l|}
\hline \multicolumn{1}{|c|}{ A nálisis } & \multicolumn{1}{|c|}{ Resultado } & \multicolumn{1}{c|}{ M étodo } \\
\hline Conductividad elect. & $0.98 \mathrm{mmh} / \mathrm{cm}$ & \\
A rena & $38 \%$ & \\
Limo & $28 \%$ & \\
A rcilla & $34 \%$ & \\
Textura & A reno-A rcilloso & Higrómetro \\
pH & 4.3 & Potenciómetro \\
CO3 Ca & $0.0 \%$ & Gaso volumétrico \\
M ateria orgánica & $0.9 \%$ & W alkey y Blas \\
Nitrógeno & $0.12 \%$ & M icro K jeldahl \\
Fósforo disponible & $5.9 \mathrm{ppm}$ & Olsen modif.. \\
K20 & $188.0 \mathrm{~kg} / \mathrm{ha}$ & Peech \\
Ca & $3.6 \mathrm{me} / 100 \mathrm{~g}$ & E.D.T.A. \\
M g & $0.8 \mathrm{me} / 100 \mathrm{~g}$ & A marillo tiazol \\
K & $0.16 \mathrm{me} / 10 \mathrm{~g}$ & Fotometro llama \\
$\mathrm{Na}$ & $0.06 \mathrm{me} / 100 \mathrm{~g}$ & Fotometro llama \\
A luminio cambiable & $4.89 \mathrm{me} / 100 \mathrm{~g}$ & \\
\hline \multicolumn{2}{|l|}{} \\
\hline
\end{tabular}

\section{APLICACIÓN DEL HL Y MANEJO DE LA PLANTACIÓN DE BOLAINA}

La bolaina usada en este estudio tuvo en promedio $31 \mathrm{~cm}$ de altura, de cuatro meses de edad. Se instaló en condiciones de campo abierto. Se hicieron hoyos de $30 \times 30 \times 30 \mathrm{~cm}$ en las parcelas. En cada hoyo se aplicaron las dosis de HL mezclada con tierra de la capa superior del suelo y en el testigo se utilizó tierra proveniente del hoyo mezclada con tierra de la capa superior del suelo. Después del trasplante se realizaron riegos cada semana y podas de las plantas eliminando las ramas laterales leñosas. La estructura de los tratamientos se muestra en el cuadro 2. 


\section{Cuadro 2}

Estructura de los tratamientos en estudio

\begin{tabular}{|l|c|c|}
\hline Clave & Dosis HL & Distanciamiento \\
\hline & $\mathrm{kg}$ & $\mathrm{cm}$ \\
$\mathrm{A} 0 \mathrm{~b} 0$ & 0 & $1 \times 1$ \\
$\mathrm{~A} 0 \mathrm{~b} 1$ & 0 & $2 \times 2$ \\
$\mathrm{~A} 0 \mathrm{~b} 2$ & 0 & $3 \times 3$ \\
$\mathrm{~A} 1 \mathrm{~b} 0$ & 1 & $1 \times 1$ \\
$\mathrm{~A} 1 \mathrm{~b} 1$ & 1 & $2 \times 2$ \\
A1b2 & 1 & $3 \times 3$ \\
A2b0 & 2 & $1 \times 1$ \\
A2b1 & 2 & $2 \times 2$ \\
A2b2 & 2 & $3 \times 3$ \\
\hline
\end{tabular}

Dosis de HL (kg):

Distanciamiento de siembra $(\mathrm{cm})$ :

$$
\begin{aligned}
& \mathrm{a}_{0}=0, \mathrm{a}_{1}=1, \mathrm{a}_{2}={ }_{2} \\
& \mathrm{~b}_{0}=1 \times 1, \mathrm{~b}_{1}=2 \times 2, \mathrm{~b}_{2}=3 \times 3
\end{aligned}
$$

Las parcelas fueron de: $5 \times 10 \mathrm{~m}, 4 \times 10 \mathrm{~m}$ y $6 \times 12 \mathrm{~m}$, de forma rectangular, y trasplantadas con 50, 10, y 8 plantones de bolaina, respectivamente. El distanciamiento entre parcelas fue de $2 \mathrm{~m}$. El arreglo factorial de las combinaciones de tratamientos se presenta en el cuadro 3. Cada tratamiento se repitió dos veces. 


\section{Cuadro 3}

Arreglo Factorial de los Tratamientos

\begin{tabular}{|l|l|ccc|}
\hline & & \multicolumn{3}{|c|}{ Factor A } \\
& & a0 & a1 & a2 \\
\hline \multirow{4}{*}{ Factor B } & b0 & a0b0 & a1b0 & a2b0 \\
& b1 & a0b1 & a1b2 & a2b2 \\
& b2 & a1b2 & a1b2 & a2b2 \\
\hline
\end{tabular}

$\mathrm{A}=\mathrm{F}$ actor dosis de humus, $\mathrm{B}=\mathrm{F}$ actor distanciamiento de siembra.

Dosis de $H \mathrm{~L}(\mathrm{~kg})$ : a $0=0, a 1=1, a 2=2$

Distanciamiento de siembra $(\mathrm{cm}): \mathrm{b} 0=\mid \mathrm{x} 1, \mathrm{~b} 1=2 \times 2, \mathrm{~b} 2=3 \times 3$.

EI HL usado fue proporcionado por el CRI-IIAP-Ucayali, sus características se presentan en el Cuadro 4.

\section{Cuadro 4}

Propiedades del HL usado en el experimento, proveniente de una mezcla de estiércol de ovino/orujo.

\begin{tabular}{|c|c|c|c|c|c|c|c|}
\hline $\mathrm{PH}$ & $\mathrm{M} \mathrm{O}$ & $\mathrm{C} / \mathrm{N}$ & $\mathrm{N}$ & $\mathrm{P}$ & $\mathrm{K}$ & $\mathrm{Ca}$ & $\mathrm{Mg}$ \\
\hline & $\%$ & $\%$ & $\%$ & $\%$ & $\mathrm{Ppm}$ & $\%$ & $\%$ \\
& & & & & & & \\
6.5 & 58.37 & 13.04 & 2.60 & 0.13 & 0.0017 & 0.24 & 0.24 \\
\hline
\end{tabular}

Fuente: CRI-IIAP-U cayali. 


\section{ANÁLISIS ESTADIS TICO}

Se usó un arreglo factorial distribuido en un diseño de bloques completamente al azar generado por tres dosis de $\mathrm{HL}$, tres distanciamiento de siembra y dos repeticiones, según el modelo matemático:

$$
X i j k=u+a i+b i+(a B) i j+p k+i j k
$$

Donde:

Xijk = observación cualquiera

$u=$ efecto de la media

ai $=$ efecto del nivel i ésimo del factor $\mathrm{A}$

$B i=$ efecto del nivel $\mathrm{i}$ ésimo del factor $B$

$(\mathrm{aBO}) \mathrm{ij}=$ efecto de la interacción del nivel $\mathrm{i}$ ésimo del factor $\mathrm{A}$ con el nivel $\mathrm{j}$ ésimo del factor $B$.

pk = es el efecto del bloque k ésimo.

ijk = es un efecto al eatorio.

\section{VARIABLES DE RESPUESTA}

\section{Variables dependientes}

Se midió la altura y el diámetro de plantas para ver su incremento en función a la dosis HL y a distanciamientos diferentes.

El diámetro se midió a los 60 días del trasplante y las evaluaciones posteriores se realizaron cada 15 días. La altura inicial se midió al momento del trasplante y luego cada 15 días.

\section{Variables Independientes}

Fueron las tres dosis de HL y los tres distanciamientos de siembra. 


\section{RESULTADOS Y DISC USION}

\section{VE LOCIDAD DE CRECIMIENTO DE Guazuma crinita POR EFECTO DE TRES DOSIS DE HL, DURANTE 150 DÍAS}

La bolaina blanca registró una velocidad de crecimiento en altura de $3 \mathrm{~cm}$ cada 15 días cuando no son tratados con HL. Con dosis de 1 y $2 \mathrm{~kg}$ de $\mathrm{HL}$ Las alturas fueron de 7 y $9 \mathrm{~cm}$ (Cuadro 5).

\section{Cuadro 5}

Velocidad de crecimiento inicial de Guazuma crinita por erecto de tres dosis de HL, durante 150 días. Pucallpa, 1992-1993. Promedio de dos repeticiones.

\begin{tabular}{|c|c|c|c|}
\hline \multirow[b]{2}{*}{ Días } & \multicolumn{3}{|c|}{ D osis de humus (kg/planta) } \\
\hline & 0 & 1 & 2 \\
\hline & -- & $\cdots--C$ & $\cdots$ \\
\hline 15 & 0.1 & 2.2 & 4.5 \\
\hline 30 & 0.8 & 5.7 & 5.8 \\
\hline 45 & 2.5 & 7.3 & 8.0 \\
\hline 60 & 2.3 & 8.2 & 11.2 \\
\hline 75 & 1.0 & 3.0 & 3.2 \\
\hline 90 & 3.8 & 5.6 & 5.7 \\
\hline 105 & 5.3 & 10.1 & 18.0 \\
\hline 120 & 4.5 & 8.7 & 9.8 \\
\hline 135 & 5.2 & 10.6 & 13.9 \\
\hline 150 & 2.4 & 8.1 & 9.9 \\
\hline Total & 28.9 & 69.5 & 90.0 \\
\hline Promedio cada 15 días & 3.0 & 7.0 & 9.0 \\
\hline
\end{tabular}




\section{ALTURA DE Guazuma crinita POR EFECTO DE TRES DOSIS DE HL Y TRES DISTANCIAMIENTO DE SIEMBRA, DURANTE 150 DÍAS}

EI ANVA (Cuadro 6) mostró que los tratamientos tuvieron un efecto positivo $(p<0.05)$ en la altura de plantas. Fue relevante la influencia del $\mathrm{HL}$ (factor A) en los incrementos de altura, pero ésta no fue afectada por las distancias de siembra (Factor B). La interacción de la dosis de HL (A) por distancia de siembra (B) tampoco fue significativo.

\section{Cuadro 6}

ANVA de la altura y diámetro de Guazuma crinita por efecto de tres dosis de HL y tres distancias de siembra.

\begin{tabular}{|l|l|l|l|l|l|}
\hline \multirow{2}{*}{ FV } & \multirow{2}{*}{} & \multicolumn{2}{|c|}{ Altura } & \multicolumn{2}{c|}{ Diámetro } \\
\cline { 3 - 6 } & GL & CM & Sig & CM & \multicolumn{1}{|c|}{ Sig } \\
\cline { 3 - 6 } Trat & 8 & 1551.83 & $*$ & 0.1609 & ns \\
A & 2 & 4959.49 & $*$ & 0.2644 & $*$ \\
B & 2 & 1044.22 & ns & 0.0696 & ns \\
A B & 4 & 101.79 & ns & 0.1548 & ns \\
Bloq & 1 & 409.84 & ns & 0.1075 & ns \\
Error & 8 & 307.61 & & 0.0511 & \\
Total & 17 & & & & \\
\hline
\end{tabular}

$*$ significativo al $5 \%, \mathrm{~A}=$ dosis de $\mathrm{HL}, \mathrm{B}=$ distancia de siembra

Las comparaciones ortogonales (Cuadro 7) entre tratamientos con 1 y $2 \mathrm{~kg}$ de HL (a2 vs. al), establecen que no existe diferencia significativa entre éstas dosis de $\mathrm{HL}$, lo que permite establecer que la dosis económica y fisiológica para la bolaina blanca sería de $1 \mathrm{~kg} / \mathrm{planta}$, para ser aplicado en el momento del trasplante a campo abierto. 
A. Quevedo G.

\section{Cuadro 7}

Comparaciones ortogonales de la altura G uazuma crinita por efecto de tres dosis de $H L$.

\begin{tabular}{|c|c|c|}
\hline Comparación HL & al & Significación \\
\hline $2 \mathrm{~kg} \vee \mathrm{s} 1 \mathrm{~kg}$ & 1 & ns \\
\hline $2 \mathrm{~kg} \vee \mathrm{s} 1 \mathrm{~kg}$ & ----- Diámetro ------ & ns \\
\hline
\end{tabular}

a1, a2 = dosis de 1 y $2 \mathrm{~kg} /$ planta de $\mathrm{HL}$.

\section{DIÁMETRO DE Guazuma crinita POR EFECTO DE TRES DOSIS DE HL Y TRES DISTANCIAMIENTOS DE SIEMBRA, DURANTE 150 DÍAS}

EI ANVA del diámetro de plantas mostró que éste no fue afectado por los tratamientos aplicados. Sin embargo, las dosis de $\mathrm{HL}$ si tuvieron efecto ( $p$ $<0.05)$. La interacción A x B tampoco fue significativo.

Al igual que en la altura de plantas, las comparaciones ortogonales muestran que el diámetro de plantas fue influenciado por la dosis de HL (Factor A) (Cuadro 7), pero no lo fue por la distancia de siembra (Factor B). Esto demuestra que al utilizar $\mathrm{HL}$ en plantaciones de bolaina blanca se favorece el crecimiento del diámetro.

CRE CIMIENTO EN ALTURA DE Guazuma crinita POR EFECTO DE TRES DOSIS DE HL Y TRES DISTANCIAMIENTOS DE SIEMBRA, DURANTE 150 DÍAS

Los mayores crecimientos iniciales en altura, durante 150 días, se observaron en los tratamientos a2b0 ( $2 \mathrm{~kg}$ de $\mathrm{HL}$ y $1 \times 1 \mathrm{~m}$ de distanciamiento) con incrementos de $95 \mathrm{~cm}$ y albo (1 kg de y $1 \times 1 \mathrm{~m}$ ) con $90 \mathrm{~cm}$ (Cuadro 8). El diámetro de $G$. crinita obtuvo un incremento final de 0.88 y $0.83 \mathrm{~cm}$ en los tratamientos a2b0 y alb0, respectivamente (Cuadro 8). 


\section{Cuadro 8}

\section{Incremento en altura y diámetro por efecto de tratamientos en $\mathbf{G}$ uazuma crinita, a los 150 días del transplante. Promedio de dos repeticiones.}

\begin{tabular}{|c|c|c|c|c|c|c|c|}
\hline & & Comb & ión fact & de trata & entos & & \\
\hline $\begin{array}{l}\mathrm{a} 0 \mathrm{~b} 0 \\
\mathrm{a} 2 \mathrm{~b} 2\end{array}$ & a0b1 & a0b2 & albo & alb1 & alb2 & albo & $\mathrm{a} 2 \mathrm{~b} 1$ \\
\hline $\begin{array}{l}43.6 \\
72.8\end{array}$ & $\begin{array}{l}20.0 \\
85.2\end{array}$ & 23.0 & $\begin{array}{c}\text { A ltura } \\
89.8\end{array}$ & 65.0 & 54.2 & 95.1 & \\
\hline $\begin{array}{l}0.47 \\
0.58\end{array}$ & $\begin{array}{l}0.16 \\
0.68\end{array}$ & 0.26 & $\begin{array}{c}0 \text { iáme } \\
0.83\end{array}$ & 7) 0.60 & 0.44 & 0.88 & \\
\hline
\end{tabular}

Dosis de $H L(k g): a 0=0, a 1=1, a 2=2$

Distanciamiento de siembra $(\mathrm{cm}): \mathrm{b} 0=\mid \times 1, b 1=2 \times 2, b 2=3 \times 3$

\section{CONCLUSIONES}

En base a los resultados de este experimento se concluye que:

1. La velocidad de crecimiento cada 15 días fue de $7 \mathrm{~cm}$ en la altura de $\mathrm{G}$. crinita, por efecto del uso de $\mathrm{HL}$ en el trasplante a campo abierto, durante los primeros 150 días.

2. La bolaina blanca G. crinita alcanzó un incremento de $305 \%$ de altura por efecto de la aplicación de $1 \mathrm{~kg} / \mathrm{planta}$ de $\mathrm{HL}$, durante 150 días, trasplantadas a campo abierto en un suelo de Pucallpa.

3. A los 150 días después del trasplante a campo abierto y con aplicación de $\mathrm{HL}$, el distanciamiento de siembra de $1 \times 1,2 \times 2,3 \times 3 \mathrm{~cm}$, en G. crinita, no tuvo efecto ni en su altura ni en su diámetro. 


\section{BIBLIOGRAFIA}

AROSTEGUI, A. Y SATO. 1970. “Estudio de las propiedades Físico-M ecánicas de la Madera de 16 Especies Forestales del Perú". Revista Forestal del Perú N 01 -2Limapág. 112.

BA N CO A GRARIO, 1987. M anual de Instrucciones para L ombricultura. D pto. de Divulgación Técnica. Lima- Perú pág. 44

CALLE, C.A. 1991. "Efecto del humus de Lombricultura en Pepino Cucumis sativus, Ají dulce Capsicum annum y chiclayo verdura Vigna sinensis en suelos Degradados de Pucallpa". Tesis para optar el Título de Ingeniero A grónomo. UNU - Pucallpa.

CARRERA, G.F. 1981. Experiencias y resultados de las plantaciones forestales en la Zona Forestal Alexander Von Humboltd. Documento de trabajo № 05 CENFOR XII - Pucallpa- Perú. pág. 79.

DE LAS CASAS, G. 1983. Aspectos sobre la Reforestación y el Balance Nutricional del Sitio en los Trópicos, Revista Forestal Latinoamericana $\mathrm{N}$ 2/84, IFLA, M érida - V enezuela pág. 5.

ENCARNACION, E.F. 1983. "Nomenclatura de Especies Forestales". LimaPerú. pág. 149.

FERRUZI, C. 1987. M anual de L ombricultura. Ediciones M undiprensa -M adrid. $130 \mathrm{pp}$.

GEILFUS, F. 1989. "El Arbol al Servicio del Agricultor. Manual de A groforestería para el Desarrollo Rural. Guía de Especies № 2. ENDA Caribe - Cate. Sto. Domingo. R.D. pág. 778.

GUERRA, J.C. 1993. "Efecto Residual de Humus de Lombricultura en un suelo degradado y en el rendimiento de Culantro (Coriandrum sativum)". Tesis para optar el Título de Ing. Agron. Universidad Nacional de U cayali. Pucallpa- Perú. 40 pp.

GONZALES, F.R. "Preservación y Secado de Madera de 5 Especies Forestales de la Zona de Pucallpa". Revista Forestal del Perú № 1- 2 pág. 108. 
HOSTETTLER, M. 1990. Inventario de las Reforestaciones en el ámbito de Influencia de Pucallpa. Temas Forestales pág. 66

PROYECTO CAPACITACIÓN, EXTENSIÓN Y DIVULGACIÓN FORESTAL. 1989. "M anual de Identificación de Especies Forestales". COTESU/U nidad A graria- U cayali pág. 211

QUEVED0, A. 1991. "Efecto del Humus de lombriz en Plantones de Cedrela odorata, atacados por Hypsiphylla sp. en Plantaciones a Campo A bierto". Tesis para optar el Título de Ingeniero F orestal, U NA P - Iquitos. pág. 45

RIOS, O.Z., R. RIME. 1992. Informe del Proyecto Hortalizas. Instituto de Investigaciones de la Amazonía Peruana (IIAP). Pucallpa, Perú. 10 p. (M imeografiado)

RIOS, O.Z., C.A. CALLE y R. RIME. 1993. Humus de lombricultura y su efecto en pepinillo (Cucumis sativum), ají dulce (Capsicum annum), y chiclayo verdura (Vigna sinensis), en un suelo de Pucallpa. Instituto de Investigaciones de la Amazonía Peruana (IIAP). Pucallpa, Perú. 12 p. (A rículo científico en prensa)

RIVERA, P. 1992. "Efecto de Calidades de Humus de Lombricultura en la producción de "pepino" Cucurnis sativus, L. en Suelo Degradado de Pucallpa". Tesis para optar el título de Ing. A gron. U niversidad N acional de U cayali. Pucallpa-Perú. 60 pp.

SABAC - CHILE. 1987. Centro de Desarrollo de L ombricultura. Folleto Chile.

ZAPATA, F. Y VILLAGARCIA S. 1982 M anual de uso de Fertilizantes. UNA. Programa de A gronomía. D pto. de suelos y Geología. Lima pág. 48. 